\title{
A DIRECT PROOF OF PORCELLI'S CONDITION FOR WEAK CONVERGENCE
}

\author{
R. B. DARST ${ }^{1}$
}

Effective representations of the general bounded linear functional on a Banach space $B$ play a prominent role in functional analysis for reasons among which is a quest for efficient determinative conditions for weak convergence of a sequence of elements of $B$. In spaces with a variation type norm, for example $(b v)$, the space of functions $f$ of bounded variation on the interval $[0,1]$ with $f(0)=0$, and $H(S)$, the space of bounded and finitely additive set functions on an algebra $S$ of subsets of a set $X$, such representations are of ten lacking. Nevertheless, both $(b v)$ and $H(S)$ can be mapped isomorphically and semiisometrically by well known mappings onto subspaces of appropriate $(M)$-spaces. These embeddings yield necessary and sufficient conditions for weak convergence to zero [1, Théorème 5 , p. 219]. Moreover, since the $(L)$-spaces $(b v)$ and $H(S)$ are sequentially weakly complete [3, Theorem 12], conditions for sequential weak convergence follow.

S. Leader [4, Theorem 16] showed that the following $L_{1}$-type conditions of Lebesgue are necessary and sufficient in order that a sequence $\left\{\mu_{k}\right\}$ of elements of $H(S)$ converge weakly.

(1) The sequence $\left\{\mu_{k}(E)\right\}$ converges for each $E$ in $S$.

(2) The sequence $\left\{\mu_{k}\right\}$ is equi-absolutely continuous with respect to the element $\phi$ of $H(S)$ defined by

$$
\phi(E)=\sum_{k=1}^{\infty} 2^{-k}\left(1+\left|\mu_{k}\right|(X)\right)^{-1}\left|\mu_{k}\right|(E),
$$

where $\left|\mu_{k}\right|$ is the variation of $\mu_{k}$.

P. Porcelli [5]-[7] established that the following condition

$$
\lim _{\boldsymbol{k}}\left(\sum_{i=1}^{\infty}\left|\mu_{k}\left(E_{i}\right)\right|\right)=0 \text { for each sequence }\left\{E_{i}\right\}
$$

of pairwise disjoint elements of $S$, is necessary and sufficient for weak convergence of the sequence $\left\{\mu_{k}\right\}$ to zero. He first showed the equivalent result for $(b v)$ by a "somewhat tedious" argument and then embedded $\left\{\mu_{k}\right\}$ in to $(b v)$.

Received by the editors December 7, 1965.

1 Supported by National Science Foundation Grant GPO3515. 
T. H. Hildebrandt [2] then showed the following, perhaps, even easier to apply condition

$$
\lim _{k}\left(\sum_{i=1}^{\infty} \mu_{k}\left(E_{i}\right)\right)=0 \text { for each sequence }\left\{E_{i}\right\}
$$

of pairwise disjoint elements of $S$, to be equivalent to condition (A). Related matters are discussed extensively in [5]-[7]

Our purpose is to give a relatively short direct proof of the following

Theorem. Condition (A) implies condition (2).

Since a sequence of elements of $(b v)$ can be mapped into $H(S)$ for a suitable choice of $S$, this direct proof leads to the corresponding result for $(b v)$ in a manner which avoids becoming embroiled in the topology of $[0,1]$. Moreover, since condition (B) follows easily from Banach's condition [1], we obtain the following result of P. Porcelli.

COROLLARY. The sequence $\left\{\mu_{k}\right\}$ converges weakly to zero if, and only if, condition (A) is satisfied.

Turning now to a proof of the theorem, we first state the following elementary consequences of condition (A).

LEMMA 1. If condition (A) is satisfied, $\left\{E_{i}\right\}$ is a sequence of pairwise disjoint elements of $S$, and $\epsilon>0$, then there exists a positive integer $j$ such that $\sum_{i \geq j}\left|\mu_{k}\left(E_{i}\right)\right|<\epsilon, k=1,2, \cdots$.

LEMMA 2. If condition (A) is satisfied, $\left\{F_{i}\right\}$ is a decreasing sequence of elements of $S$, and $\epsilon>0$, then there exists a positive integer $j$ such that $\sum_{i \geqq i}\left|\mu_{k}\left(F_{i}-F_{i+1}\right)\right|<\epsilon, k=1,2, \cdots$.

Proof of Theorem. Let us say that $(k, E)$ is a pair for $(\delta, \epsilon)$, $\delta>0, \epsilon>0$, if $\phi(E)<\delta$ and $\left|\mu_{k}(E)\right| \geqq \epsilon$. Let $\delta(k, \epsilon)$ be a positive number such that $\phi(E)<\delta(k, \epsilon)$ implies that $\left|\mu_{j}(E)\right|<\epsilon, j=1,2, \cdots, k$. Suppose condition (2) is not satisfied. Then there is a positive number $\boldsymbol{\epsilon}$ such that for each positive number $\delta$ there is a pair $(k, E)$ for $(\delta, 2 \epsilon)$. Let $\left(k_{1}, E_{1}\right)$ be a pair for, say, $(1,2 \epsilon)$. Let $\left(k_{2}, E_{2}\right)$ be a pair for $\left(\delta\left(k_{1}, \epsilon \cdot 2^{-2}\right), 2 \epsilon\right)$ and, proceeding inductively, let $\left(k_{i+1}, E_{i+1}\right)$ be a pair for $\left(\delta\left(k_{i}, \epsilon \cdot 2^{-(i+1)}\right), 2 \epsilon\right)$. At this point, let's relabel the sequence $\left\{\mu_{k_{i}}\right\}$ as $\left\{\mu_{i}\right\}$ and record what we have obtained thus far:

(i) $\left|\mu_{i}\left(E_{i}\right)\right| \geqq 2 \epsilon$ and

(ii) if $E \subset E_{i}$, then $\left|\mu_{j}(E)\right|<\epsilon / 2^{i}, j=1,2, \cdots, i-1$.

Let $F_{1}=E_{1}$. If there exists an integer $i$ greater than one such that $\left|\mu_{i}\left(F_{1} \cap E_{i}\right)\right|>\epsilon / 2$, let $i_{1}$ be the least such in teger and let $F_{2}=F_{1}-E_{i_{1}}$. Then if there exists an integer $i$ greater than $i_{1}$ such that $\left|\mu_{i}\left(F_{2} \cap E_{i}\right)\right|$ 
$>\epsilon / 2$, let $i_{2}$ be the least such integer and let $F_{3}=F_{2}-E_{i_{2}}$. If this process were not to stop, we would obtain, in contradiction to Lemma 2 , a decreasing sequence $\left\{F_{p}\right\}$ of elements of $S$ such that $\mid \mu_{i_{p}}\left(F_{p}\right.$ $\left.-F_{p+1}\right)|=| \mu_{i_{p}}\left(F_{p} \cap E_{i p}\right) \mid>\epsilon / 2$. Hence, there exist least positive in tegers $j_{1}$ and $p_{1}$ such that if $i>p_{1}$ then $\left|\mu_{i}\left(F_{j_{1}} \cap E_{i}\right)\right| \leqq \epsilon / 2$. In order to simplify what follows, we denote by $H_{1}$ the set $F_{j_{1}}$ of the preceding sentence, let $\mu_{i}^{\prime}=\mu_{p_{1}+i}$ and let $E_{i}^{\prime}=E_{p_{1}+i}-H_{1}$. Then

(iii) $\left|\mu_{1}\left(H_{1}\right)\right| \geqq 2 \epsilon-\epsilon / 2$,

(iv) $\left|\mu_{i}^{\prime}\left(E_{i}^{\prime}\right)\right| \geqq 2 \epsilon-\epsilon / 2$, and

(v) $\left|\mu_{j}^{\prime}(E)\right|<\epsilon / 2^{p_{1}+i} \leqq \epsilon / 2^{(i+1)}$ if $E \subset E_{i}^{\prime}$ and $j<i$.

Let $F_{1}^{\prime}=E_{1}^{\prime}$. Proceeding as before, there exist least positive integers $j_{2}$ and $p_{2}$ such that if $i>p_{2}$, then $\left|\mu_{i}^{\prime}\left(F_{j_{2}}^{\prime} \cap E_{i}^{\prime}\right)\right|<\epsilon / 2^{2}$ and $\left|\mu_{1}^{\prime}\left(F_{j_{2}}^{\prime}\right)\right| \geqq 2 \epsilon-\epsilon / 2-\epsilon / 2^{2}$. Denoting by $H_{2}$ the set $F_{j_{2}}^{\prime}$, letting $E_{i}^{2}=E_{p_{2}+i}^{\prime}-H_{2}$ and $\mu_{i}^{2}=\mu_{p_{2}+i}^{\prime}$ we obtain

(vi) $H_{1} \cap H_{2}=\varnothing$,

(vii) $\left|\mu_{p_{1}+1}\left(H_{2}\right)\right| \geqq 2 \epsilon-\epsilon / 2-\epsilon / 2^{2}$,

(viii) $\left|\mu_{i}^{2}\left(E_{i}^{2}\right)\right| \geqq 2 \epsilon-\epsilon / 2-\epsilon / 2^{2}$, and

(ix) $\left|\mu_{j}^{2}(E)\right|<\epsilon / 2^{p_{1}+p_{2}+i}<\epsilon / 2^{i+2}$ if $E \subset E_{i}^{2}$ and $j<i$.

Thus, leading next to a set $H_{3}$ such $H_{3} \cap\left(H_{1} \cup H_{2}\right)=\varnothing$, and $\left|\mu_{p_{1}+p_{2}+1}\left(H_{3}\right)\right| \geqq 2 \epsilon-\epsilon / 2-\epsilon / 2^{2}-\epsilon / 2^{3}$ and eventually, letting $q_{i}=p_{1}$ $+p_{2}+\cdots+p_{i}+1$, to a sequence $\left\{H_{i}\right\}$ of pairwise disjoint elements of $S$ such that $\left|\mu_{q_{i}}\left(H_{i+1}\right)\right|>\epsilon$ which implies that if condition (2) is not satisfied, then condition (A) is not satisfied.

\section{BIBLIOGRAPHY}

1. S. Banach, Théorie des opérations lineaires, Chelsea, New York, 1955.

2. T. H. Hildebrandt, On a theorem in the space $l_{1}$ of absolutely convergent sequences with applications to completely additive set functions, Math. Research Center Rep. No. 62, Madison, Wis., 1958.

3. S. Kakutani, Concrete representations of abstract $(L)$-spaces and the mean ergodic theorem, Ann. of Math. 42 (1941), 523-537.

4. S. Leader, The theory of $L^{p_{-}}$spaces for finitely additive set functions, Ann. of Math. 58 (1953), 528-543.

5. P. Porcelli, On weak convergence in the space of functions of bounded variation, Math. Research Center Rep. No. 39, Madison, Wis., 1958.

6. - On weak convergence in the space of functions of bounded variation. II, Math. Research Center Rep. No. 68, Madison, Wis., 1958.

7. - Two embedding theorems with applications to weak convergence and compactness in spaces of additive type functions, J. Math. Mech. 9 (1960), 273-292.

Purdue University 